\title{
Development and Validation of a Voltammetry Method to Determine Traces of Iron in Water and other Matrixes
}

\author{
Ana L. Alvarado-Gámez*, Jorge Campos-Fernández \\ Electrochemistry and Chemical Energy Center and School of Chemistry, \\ University of Costa Rica, P.O. Box 2060, San Pedro de Montes de Oca, San José, Costa Rica
}

Received 31 May 2004; accepted in revised form 21 September 2004

\begin{abstract}
It was developed a sensitive analytical methodology to determine iron in water samples and other matrixes. It was used a differential pulse cathodic stripping voltammetry technique and adsorptive preconcentration with $\mathrm{KSCN}$ as a ligand and $\mathrm{NaNO}_{2}$ as a catalytic agent. The methodology has a linear range between $0.7 \mu \mathrm{g} / \mathrm{L}$ and $10.0 \mu \mathrm{g} / \mathrm{L}$, a detection limit of $0.2 \mu \mathrm{g} / \mathrm{L}$ and a quantification limit of $0.7 \mu \mathrm{g} / \mathrm{L}$. The averaged sensitivity is $344 \mathrm{nA} /(\mu \mathrm{g} / \mathrm{L})$. The accuracy has a recovery of $102 \%$ for $1.0 \mu \mathrm{g} / \mathrm{L}$ of iron with a variation coefficient of $4 \%$. Also it was studied the linearity of the Fe-cathecol system, which has an average sensitivity of $4 \mathrm{nA} /(\mu \mathrm{g} / \mathrm{L})$, much lower than the one of the Fe-KSCN system, and a wider linear range from $2.4 \mu \mathrm{g} / \mathrm{L}$ to $50.0 \mu \mathrm{g} /$, a detection limit of $0.7 \mu \mathrm{g} / \mathrm{L}$ and quantification limit of $2.4 \mu \mathrm{g} / \mathrm{L}$, with a variation coefficient of $8 \%$ and recoveries near $100 \%$ at different concentration levels.
\end{abstract}

Keywords: adsorptive voltammetry, iron, thyocianate, nitrite, cathecol, trace analysis.

\section{Introducción}

Las técnicas voltamperométricas basadas en la formación de amalgamas de metales en un microelectrodo de gota suspendida de mercurio, permiten analizar metales como el $\mathrm{Pb}, \mathrm{Cd}, \mathrm{Cu}$ y $\mathrm{Zn}$ entre otros, con alta sensibilidad, selectividad y límites de detección y cuantificación en el orden de los $\mu \mathrm{g} / \mathrm{L}$ o décimas de éstos [1-3]. En el caso de los elementos de transición como Cr, Fe, Mo, Ni, y Co, por ejemplo, que no forman amalgamas con el mercurio a los potenciales de trabajo

\footnotetext{
* Corresponding author. E-mail address: agamez@cariari.ucr.ac.cr
} 
de este electrodo, debido a que sus potenciales de descomposición son más positivos que el del mercurio, no se pueden analizar en forma directa. Por tal motivo, en el área del electroanálisis se han desarrollado muchas técnicas voltamperométricas analíticas que conllevan procedimientos de preconcentración adsortiva, con la formación de un complejo que se adsorbe en la gota de mercurio, haciendo al metal más electroactivo, y algunas veces como ocurre con el $\mathrm{Cr}, \mathrm{Fe}$ y $\mathrm{Ni}$ se pueden utilizar reacciones catalíticas que mejoran la sensibilidad del método de análisis. Esta técnica adsortiva por tanto es complementaria de las técnicas voltamperométricas, para analizar metales a nivel de trazas y ultra trazas que son imposibles o muy difíciles de analizar por esas técnicas.

Entre los ligandos que se utilizan para acomplejar el hierro y analizarlo por voltamperometría adsortiva se encuentran el catecol, el tiocianato-óxido nítrico, tiocianato-nitrito, el violeta de solocromo, el 1-nitro-2-naftol, la 2-(5-bromo-2piridilazo)-5-dietilaminofenol, la trietanolamina, la ortofenantrolina y otros que se mencionan en la literatura [4-9].

Se desarrolló un método voltamperométrico adsortivo para analizar hierro en aguas y otras matrices, a nivel de los $\mu \mathrm{g} / \mathrm{L}$ utilizando el sistema $\mathrm{KSCN}-\mathrm{NaNO}_{2}$, como ligando y catalítico respectivamente, en medio de ácido acético, a partir del método desarrollado por Gao y Siow [6], además se validó otro método utilizando catecol como ligando [4], el cual resultó ser adecuado para analizar hierro en concentraciones más altas, siendo éste un método menos sensible que el de Fe-SCN.

\section{Parte experimental}

Toda la cristalería utilizada se lavó en baños de $\mathrm{HNO}_{3}$ calidad para análisis al $20 \% \mathrm{v} / \mathrm{v}$, por dos semanas; el agua que se utilizó en la preparación de disoluciones, enjuague de la cristalería y dilución de muestras, proviene de un sistema desionizador Barnstead y un purificador Milli Q. El equipo utilizado es un analizador Park 384 B y un sistema de electrodos EG\&G, con un microelectrodo de gota suspendida de mercurio como electrodo de trabajo, un 
electrodo de alambre de platino como electrodo auxiliar y un electrodo de referencia de $\mathrm{Ag} / \mathrm{AgCl}$. Se utilizó la técnica de adición estándar para la cuantificación, utilizando disoluciones patrón de hierro como $\mathrm{FeCl}_{3}$ preparadas a partir de una madre de $4000 \mathrm{mg} / \mathrm{L}$, proveniente de un titrisol Merck.

Se utilizó una disolución de $\mathrm{KSCN}$ como ligando y de $\mathrm{NaNO}_{2}$ como agente catalítico, y el catecol, se prepararon con reactivos calidad para análisis. El ácido acético, lo mismo que el acetato de sodio para la preparación de las disoluciones amortiguadoras fueron calidad ultrapura. Se optimizaron los parámetros instrumentales como potencial inicial y final, velocidad de barrido, altura de pulso, tiempo de depósito y de equilibrio. También se determinaron las concentraciones del ligando y del catalítico más adecuadas. Además de los parámetros de desempeño del método como ámbito lineal, límites de detección y cuantificación según Taylor [10], la precisión en términos de coeficiente de variación utilizando siete réplicas independientes de una adición de hierro de 1 $\mu \mathrm{g} / \mathrm{L}$ y se obtuvo la veracidad en términos de \% de recuperación.

\section{Resultados y discusión}

Se probaron tres ligandos para el hierro: 1,10 fenantrolina, tiocianato de potasio y catecol. Los complejos hierro-catecol y hierro-tiocianato dieron resultados positivos con el electrodo de gota suspendida de mercurio en cuanto a corriente de pico y linealidad en la cuantificación, utilizando la técnica voltamperométrica de despojo con barrido anódico y catódico, pues la reacción de adsorción de los complejos sobre el electrodo de gota de mercurio es reversible. Las mejores señales se dan en el sentido catódico. Además se empleó la preconcentración adsortiva del complejo Fe-SCN${ }^{-}$en presencia de $\mathrm{NO}_{2}{ }^{-}$, el cual ha resultado ser un buen catalítico para el proceso de transferencia de carga. Cuando se trabajó el Fe con KSCN se obtuvo una señal de corriente de $7.70 \mathrm{nA}$ para $20 \mu \mathrm{g} / \mathrm{L}$ de $\mathrm{Fe}$, en ausencia de nitrito y de $1512 \mathrm{nA}$ en $0.01 \mathrm{~mol} / \mathrm{L}$ de $\mathrm{NaNO}_{2}$, lo que significa que la señal aumenta con el nitrito en forma significativa. El pico de Fe-catecol es bien definido pero la sensibilidad es mucho más baja que en el sistema Fe-SCN . El sistema hierro-ortofenantrolina a pesar de que presentó una señal en $-0,750 \mathrm{~V}$, el 
pico corresponde a la adsorción del ligando y el mismo disminuye conforme aumenta la adición de $\mathrm{Fe}$, por lo que se consideró poco preciso el utilizar corrientes que disminuyen con el aumento de concentración del elemento de interés.

Se decidió trabajar con el $\mathrm{SCN}^{-}$como ligando y se establecieron las condiciones instrumentales de la técnica de análisis con la técnica voltamperométrica de despojo catódico con pulso diferencial y preconcentración adsortiva.

A

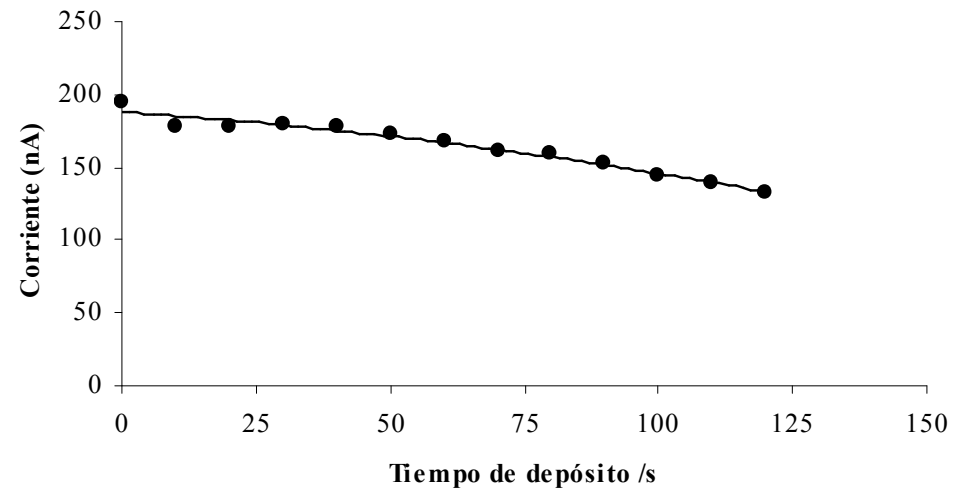

B

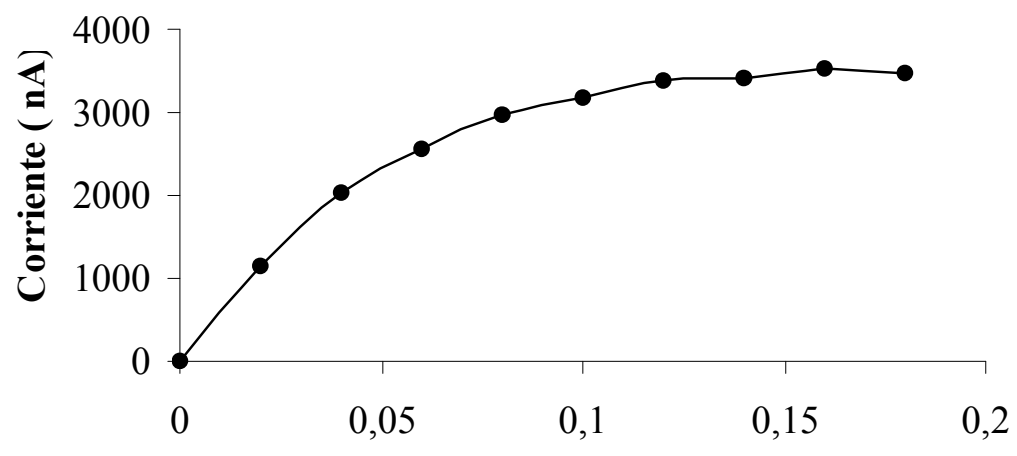

Concentración de $\mathrm{NaNO}_{2}(\mathrm{mg} / \mathrm{L})$

Figura 1. Estudio del efecto del tiempo de depósito (A), y el efecto de la velocidad de barrido (B) sobre la señal de $3 \mu \mathrm{g} / \mathrm{L}$ de $\mathrm{Fe}^{3+}$, en presencia de $0.2 \mathrm{~mol} / \mathrm{L}$ de $\mathrm{HOAc}, 0.1$ $\mathrm{mol} / \mathrm{L}$ de $\mathrm{NaNO}_{2}$ y $0.03 \mathrm{~mol} / \mathrm{L}$ de $\mathrm{KSCN}$. 
Se estudiaron los parámetros de desempeño del método con tiocianato de potasio como agente acomplejante y nitrito de sodio como catalítico utilizando reactivos suprapuros. Se establecieron las concentraciones óptimas de cada uno de los reactivos a utilizar en la celda polarográfica: $\mathrm{KSCN} 0.02 \mathrm{~mol} / \mathrm{L}, \mathrm{NaNO}_{2} 0.03$ $\mathrm{mol} / \mathrm{L}$ y $\mathrm{CH}_{3} \mathrm{COOH} 0.2 \mathrm{~mol} / \mathrm{L}$.

Se fijaron todos los parámetros inicialmente basándose en los establecidos por Gao y Siow [3] y se fue variando uno a uno hasta obtener la mejor señal de corriente. Por ejemplo, como se detalla en las Fig. 1A y 1B, se varió el tiempo de depósito desde 0 hasta 125 segundos y la señal de $\mathrm{Fe}^{3+}$ fue disminuyendo, pues conforme pasa el tiempo, la gota se va saturando. El tiempo óptimo resultó ser de 10 segundos, pues la gota no se satura como ocurre con tiempos largos de depósito, además se obtiene una buena señal y sensibilidad adecuada. El tiempo de equilibrio de 10 segundos y el de purga de 30 segundos resultaron ser los más adecuados, pues la señal de hierro decae con el tiempo y el análisis debe realizarse en forma rápida y no es recomendable tener purgas de varios minutos como en la mayoría de otros métodos. El potencial del pico está en -0,380 V vs. $\mathrm{Ag} / \mathrm{AgCl}(\mathrm{sat})$. Se varió la velocidad de barrido desde 2 hasta $20 \mathrm{mV} / \mathrm{s}$ y se puede observar en la Fig. 1B, que no hay mucha variación en la señal de Fe con la variación de la velocidad de barrido, las señales oscilaron entre $162.8 \mathrm{nA}$ para 2 $\mathrm{mV} / \mathrm{s}$ y $219.4 \mathrm{nA}$ para $6 \mathrm{mV} / \mathrm{s}$. Sin embargo, se escogió una velocidad de barrido de $10 \mathrm{mV} / \mathrm{s}$, con un incremento de barrido de $5 \mathrm{mV}$ y un tiempo de gota de 0,5 segundos, por tener mejor sensibilidad cuando se corrieron curvas de adición estándar, para un tiempo de electrodepósito de 5 segundos, como se demuestra en la tabla 1. Esto se contrapone a lo reportado por Gao y Siow [6] quienes recomiendan una velocidad de $100 \mathrm{mV} / \mathrm{s}$ y un tiempo de electrodepósito de 30 segundos. 
Tabla 1. Datos de las curvas de calibración a diferentes velocidades de barrido para el sistema $\mathrm{Fe} / \mathrm{KSCN}$.

\begin{tabular}{ccc}
\hline $\begin{array}{c}\text { Velocidad de barrido } \\
\mathbf{m V} / \mathbf{s}\end{array}$ & $\begin{array}{c}\text { Sensibilidad } \\
\mathbf{n A} /(\boldsymbol{\mu g} / \mathbf{L})(\mathbf{p e n d i e n t e})\end{array}$ & $\begin{array}{c}\text { Coeficiente de correlación } \\
\mathbf{r}\end{array}$ \\
\hline 10 & 674.5 & 0.9974 \\
15 & 538 & 0.9981 \\
20 & 667 & 0.9940 \\
30 & 615.5 & 0.9953 \\
\hline
\end{tabular}

También se varió la altura del pulso desde 10 hasta $140 \mathrm{mV}$ y se observó que entre 80 y $120 \mathrm{mV}$ la variación en la altura del pulso se mantiene casi constante, por lo que se tomó como adecuado el valor medio de $100 \mathrm{mV}$, como se observa en la Fig. 2.

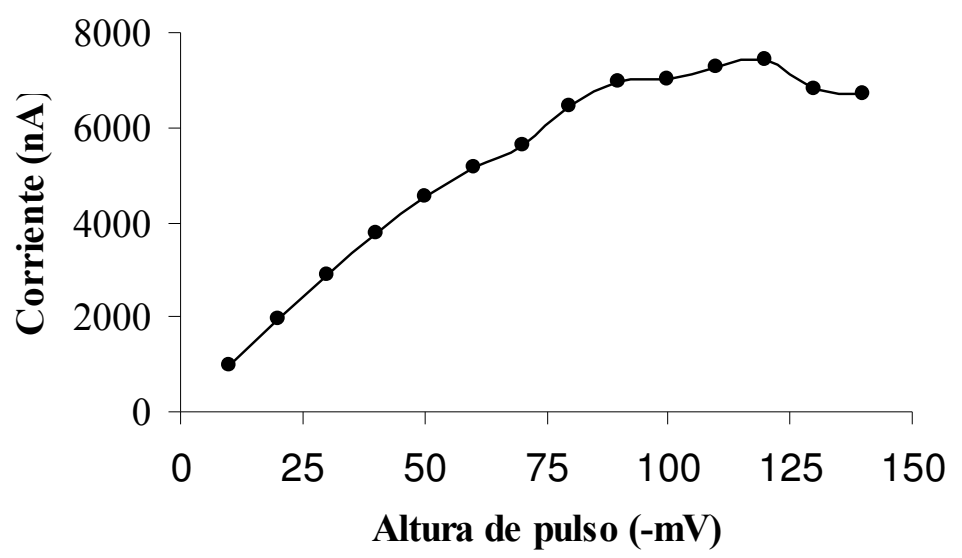

Figura 2. Estudio del efecto de la altura de pulso sobre la señal de $3 \mu \mathrm{g} / \mathrm{L}$ de $\mathrm{Fe}^{3+}$, en presencia de $0.2 \mathrm{~mol} / \mathrm{L}$ de HOAc, $0.1 \mathrm{~mol} / \mathrm{L}$ de $\mathrm{NaNO}_{2}$ y $0.03 \mathrm{~mol} / \mathrm{L}$ de $\mathrm{KSCN}$.

Seguidamente se estudiaron las concentraciones adecuadas del ligando KSCN y el catalítico $\mathrm{NaNO}_{2}$. Como se observa en la Fig. 3A, la concentración de KSCN se fue variando desde 0 hasta $0.07 \mathrm{~mol} / \mathrm{L}$ para el $\mathrm{KSCN}$ y en la Fig. 3B, se varió el catalítico desde 0 hasta $0.18 \mathrm{~mol} / \mathrm{L}$ para el $\mathrm{NaNO}_{2}$. Se recomiendan entonces las concentraciones entre 0.02 y $0.03 \mathrm{~mol} / \mathrm{L}$ para el ligando, pues a esas concentraciones se obtienen las mejores señales y de $0.1 \mathrm{~mol} / \mathrm{L}$ para el nitrito, porque a partir de esa concentración la altura de pico del Fe se mantiene casi 
constante. Se varió además el pH del medio, utilizando desde ácido acético solo hasta mezclas de ácido acético y acetato de sodio y el sistema que dio mejores resultados fue el de $\mathrm{CH}_{3} \mathrm{COOH}$ al $0.2 \mathrm{~mol} / \mathrm{L}$ en celda, pues en los $\mathrm{pH}$ mayores la señal del pico disminuye. Además se probó utilizando el medio de ácido acético y se fue adicionando $\mathrm{NaOH}$ y el comportamiento fue el mismo, conforme se agrega más base, la señal del Fe fue disminuyendo.

A

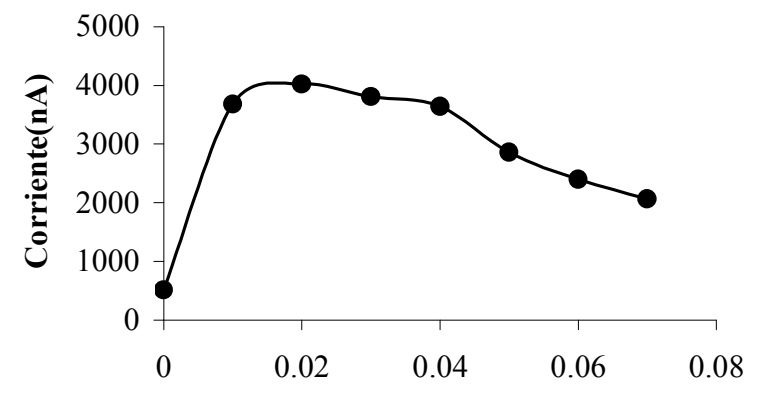

Concentración de $\mathrm{SCN}^{-}(\mathrm{mol} / \mathrm{L})$

B

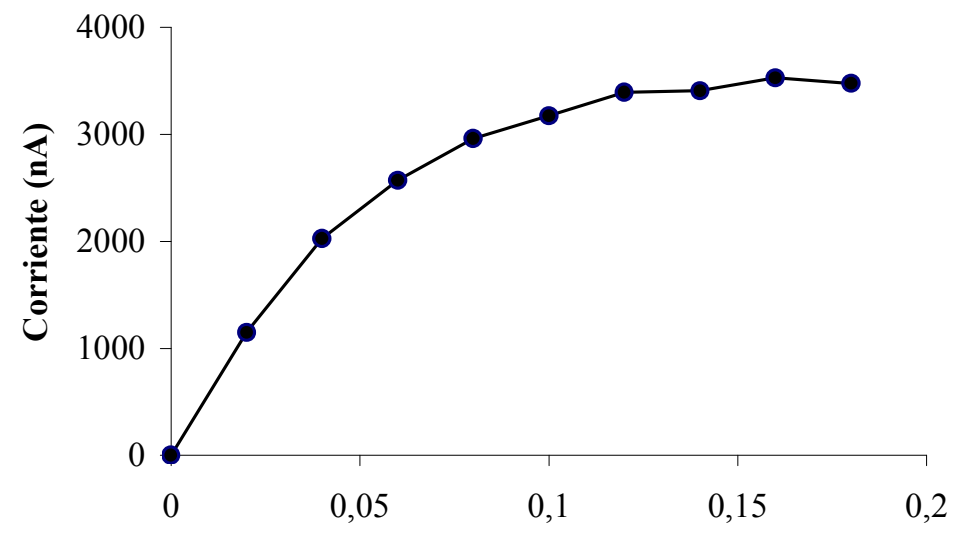

Concentración de $\mathrm{NaNO}_{2}(\mathrm{~mol} / \mathrm{L})$

Figura 3. Efecto de la concentración de $\mathrm{KSCN}$ como ligando (A) y de $\mathrm{NaNO}_{2}$ como catalítico (B) sobre la señal de $3 \mu \mathrm{g} / \mathrm{L}$ de $\mathrm{Fe}^{3+}$, en presencia de $0.2 \mathrm{~mol} / \mathrm{L}$ de $\mathrm{HOAc}$, tiempo de depósito $10 \mathrm{~s}$, velocidad de barrido de $10 \mathrm{mV} / \mathrm{s}$ y una altura de pulso de 100 $\mathrm{mV}$. 
Se determinó el ámbito lineal del método $\mathrm{Fe} / \mathrm{KSCN}$ y se encontró que la linealidad se da entre 1 y $10 \mu \mathrm{g} / \mathrm{L}$. En la Fig. 4 se muestra una de las curvas de adición estándar para Fe.

Otros parámetros de desempeño del método que se determinaron fueron los límites de detección y cuantificación, a partir de blancos preparados según lo recomienda Taylor en forma independiente, cuyos valores aparecen en la tabla 2. La sensibilidad depende de la preparación reciente del reactivo $\mathrm{NaNO}_{2}$ que se utiliza como catalítico, ya que si se utilizaba una disolución vieja la sensibilidad en $\mathrm{nA} /(\mu \mathrm{g} / \mathrm{L})$ era muy pobre: entre $62 \mathrm{nA} /(\mu \mathrm{g} / \mathrm{L})$ y $22.6 \mathrm{nA} /(\mu \mathrm{g} / \mathrm{L})$; mientras que con el reactivo fresco la sensibilidad mejora considerablemente, entre 538 y 679 $\mathrm{nA} /(\mu \mathrm{g} / \mathrm{L})$, por eso se debe cambiar la disolución todas las semanas. La sensibilidad media es $625 \mathrm{nA} / \mu \mathrm{g} / \mathrm{L}$, con una desviación estándar de 50 $\mathrm{nA} /(\mu \mathrm{g} / \mathrm{L})$.

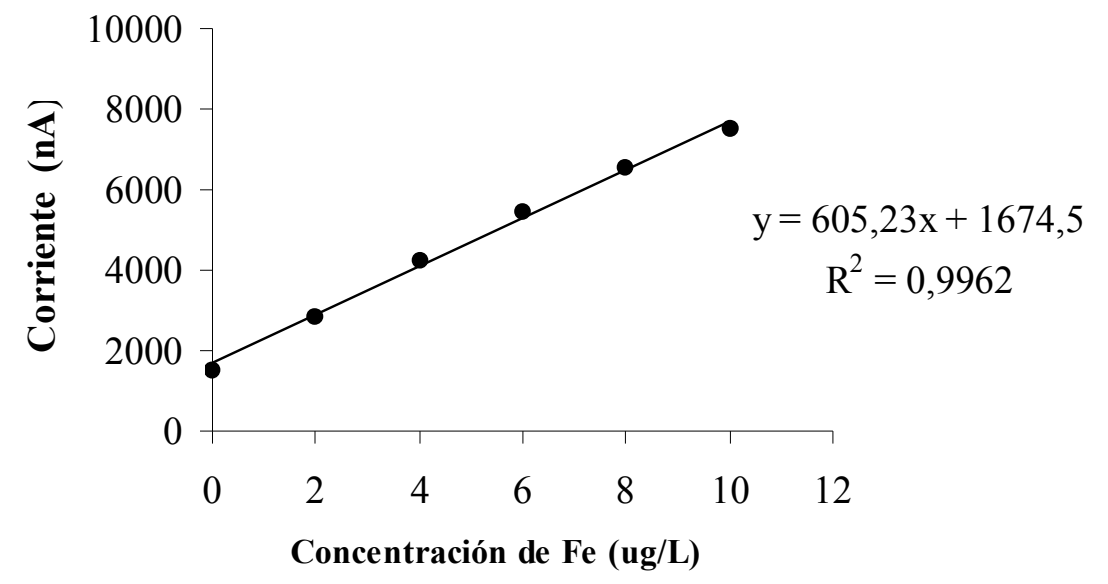

Figura 4. Curva de adición estándar para Fe en presencia de $0.2 \mathrm{~mol} / \mathrm{L}$ de $\mathrm{HOAc}, 0.1$ mol/L de $\mathrm{NaNO}_{2}$ y $0.03 \mathrm{~mol} / \mathrm{L}$ de $\mathrm{KSCN}$, tiempo de depósito $10 \mathrm{~s}$, velocidad de barrido $10 \mathrm{mV} / \mathrm{s}$ y una altura de pulso de $100 \mathrm{mV}$. 
Tabla 2. Límites de detección y cuantificación para hierro para el sistema Fe-KSCN en presencia de $\mathrm{NaNO}_{2}$.

\begin{tabular}{|c|c|c|c|c|c|}
\hline \multicolumn{3}{|c|}{ Concentración $\mu \mathrm{g} / \mathrm{L}$} & \multicolumn{3}{|c|}{ Pendiente / sensibilidad nA/ $(\mu \mathrm{g} / \mathrm{L})$} \\
\hline $\begin{array}{c}\text { Promedio } \\
7 \text { réplicas } \\
1.70\end{array}$ & $\begin{array}{c}\text { Desviación } \\
\text { estándar } \\
0.070\end{array}$ & $\begin{array}{c}\text { Coeficiente } \\
\text { de variación } \\
4.0 \%\end{array}$ & $\begin{array}{c}\text { Promedio } \\
344\end{array}$ & $\begin{array}{c}\text { Desviación } \\
\text { estándar } \\
21\end{array}$ & $\begin{array}{c}\text { Coeficiente } \\
\text { de variación } \\
6.1 \%\end{array}$ \\
\hline $\begin{array}{l}\text { Límite de } \mathrm{d} \\
\text { Límite de c }\end{array}$ & $\begin{array}{l}\text { cción } \\
\text { tificación }\end{array}$ & & & $\begin{array}{l}0.20 \mu \mathrm{g} / \mathrm{L} \\
0.70 \mu \mathrm{g} / \mathrm{L}\end{array}$ & \\
\hline
\end{tabular}

Tabla 3. Porcentajes de recuperación para $1.0 \mu \mathrm{g} / \mathrm{L}$ en $\mathrm{Fe}^{3+}$ para el sistema Fe-KSCN y $\mathrm{NaNO}_{2}$.

\begin{tabular}{cccc}
\hline Réplica & $\begin{array}{c}\text { Pendiente } \\
\text { sensibilidad } \\
\mathbf{n A} /(\boldsymbol{\mu g} / \mathbf{L})\end{array}$ & $\begin{array}{c}\text { Coeficiente } \\
\text { de correlación }\end{array}$ & $\begin{array}{c}\text { \% de recuperación } \\
\text { para } \mathbf{1} \boldsymbol{\mu g} / \mathbf{L}\end{array}$ \\
\hline Promedio & 486 & 0.9928 & 102.0 \\
S & 93 & 0.0039 & 5.7 \\
C.V. $\%$ & 19 & 0.4 & 5.6 \\
\hline
\end{tabular}

Este método se ha aplicado en la determinación de hierro en diferentes muestras. Se analizaron seis muestras por triplicado de diferentes nacientes de agua de una zona de La Cruz de Guanacaste y se probó el método desarrollado. Las concentraciones de hierro en las muestras de agua analizadas oscilaron entre detectable y $7.4 \mathrm{mg} / \mathrm{L}$. También se analizaron nueve muestras diferentes de tapas plásticas para refrescos y se utilizaron dos tipos de tratamiento: una digestión húmeda con $\mathrm{H}_{2} \mathrm{SO}_{4}$ y luego con $\mathrm{HNO}_{3}$ y $\mathrm{HClO}_{4}$; un segundo tratamiento con extracción con $\mathrm{HCl}$ concentrado. Todos los reactivos de calidad ultrapura. Todas las muestras presentaron un contenido de hierro no detectable. $\mathrm{E}$ l método se probó en vino tinto, con digestión húmeda con $\mathrm{HNO}_{3} \mathrm{y} \mathrm{HClO}_{4}$. El promedio en el vino fue de $74.2 \mu \mathrm{g} / \mathrm{L}$ con un DRP de 1.7 .

Se estudió además otro ligando, el catecol, utilizado por Van der Berg y Huang [4], el cual dio buenos resultados. De nuevo se optimizaron las condiciones de análisis de la misma manera que el caso del KSCN y la señal analítica resultó ser directamente proporcional a la concentración de hierro. Los parámetros instrumentales y los de desempeño del método se detallan en las tablas 4 y 5 , 
respectivamente. Es importante mencionar que este método que utiliza el complejo Fe-catecol tiene baja sensibilidad, como se observa en las Fig. 5A y 5B, pero tiene un amplio ámbito de linealidad, comparado con la sensibilidad del sistema Fe-KSCN, tablas 3 y 5.

Tabla 4. Parámetros instrumentales para la determinación de hierro con el sistema FeKSCN y Fe-catecol.

\begin{tabular}{|l|r|r|}
\hline Parámetros instrumentales & Fe/KSCN & Fe/catecol \\
\hline Potencial inicial vs. Ag/AgCl & $-0.200 \mathrm{~V}$ & $-0.100 \mathrm{~V}$ \\
Potencial final & $-0.700 \mathrm{~V}$ & $-0.650 \mathrm{~V}$ \\
Tiempo de depósito & $10 \mathrm{~s}$ & $180 \mathrm{~s}$ \\
Tiempo de equilibrio & $10 \mathrm{~s}$ & $10 \mathrm{~s}$ \\
Altura de pulso & $100 \mathrm{mV}$ & $100 \mathrm{mV}$ \\
Velocidad de barrido & $10 \mathrm{mV} / \mathrm{s}$ & $50 \mathrm{mV} / \mathrm{s}$ \\
Potencial de pico & $-0.380 \mathrm{~V}$ & $-0.360 \mathrm{~V}$ \\
\hline
\end{tabular}

Tabla 5. Parámetros de desempeño del método Fe-catecol, con amortiguador de acetatos a pH 6.8 y concentración de catecol $9 * 10^{-4} \mathrm{~mol} / \mathrm{L}$.

\begin{tabular}{|c|c|c|c|c|c|}
\hline \multicolumn{3}{|c|}{ Ámbito lineal para Fe } & \multicolumn{3}{|c|}{$4 \mu \mathrm{g} / \mathrm{L}$ y $50 \mu \mathrm{g} / \mathrm{L}$} \\
\hline Límite de detecc & $\mathrm{n}$ & & $1.3 \mu \mathrm{g} / \mathrm{L}$ & & \\
\hline Límite de cuanti & cación & & $4.0 \mu \mathrm{g} / \mathrm{L}$ & & \\
\hline Concentración & $\bar{n}$ & $\begin{array}{c}\text { Recuperación } \\
\% \\
\end{array}$ & $\begin{array}{c}\text { Desviación } \\
\text { estándar }\end{array}$ & C.V. \% & $\begin{array}{c}\text { Sensibilidad } \\
\mathbf{n A} /(\mu \mathrm{g} / \mathrm{L})\end{array}$ \\
\hline $4 \mu \mathrm{g} / \mathrm{L}$ & 7 & 101.5 & 3.8 & 3.8 & 4.77 \\
\hline $10 \mu \mathrm{g} / \mathrm{L}$ & 7 & 99 & 11 & 11.2 & 3.69 \\
\hline $20 \mu \mathrm{g} / \mathrm{L}$ & 5 & 112 & 7.7 & 6.9 & 3.87 \\
\hline
\end{tabular}




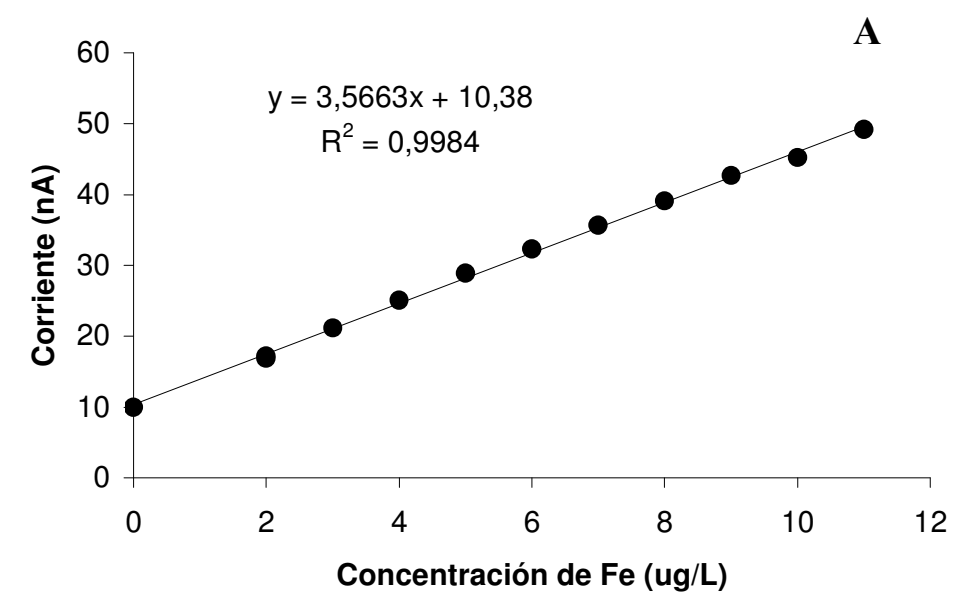

B

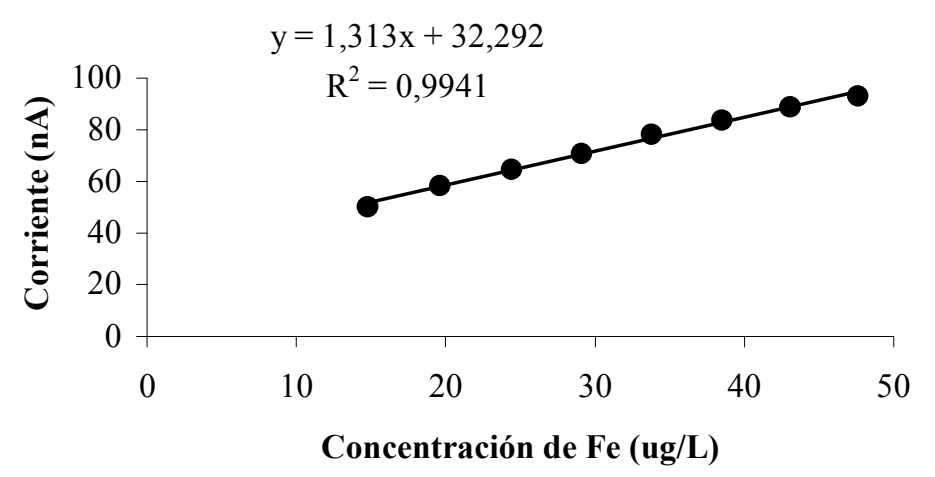

Figura 5. Curva de adición estándar para Fe en presencia de $0.0006 \mathrm{~mol} / \mathrm{L}$ de catecol, HOAc/NaOAc pH 6.8, velocidad de barrido $50 \mathrm{mV} / \mathrm{s}$ y altura de pulso $100 \mathrm{mV}$, en el ámbito de 1 a $10 \mu \mathrm{g} / \mathrm{L}$ (A) y entre $15 \mu \mathrm{g} / \mathrm{L}$ y $50 \mu \mathrm{g} / \mathrm{L}$ (B).

\section{Conclusiones}

Como conclusiones se puede mencionar que se establecieron las condiciones óptimas de dos metodologías analíticas para analizar hierro a nivel de trazas en aguas y otras muestras.

El método que utiliza el sistema $\mathrm{Fe}-\mathrm{KSCN}$ en presencia de $\mathrm{NaNO}_{2}$ resulta ser cien veces más sensible que el de Fe-catecol, además es más rápido y tiene límites de detección y cuantificación mejores. Se espera trabajar con todos los 
reactivos ultrapuros y mejorar los límites de detección y cuantificación así como la sensibilidad de este último.

\section{Agradecimientos}

Se agradece a la Vicerrectoría de Investigación, a la Escuela de Química y al Centro de Electroquímica y Energía Química (CELEQ) de la Universidad de Costa Rica por toda la colaboración y apoyo económico brindado para llevar a cabo esta investigación.

\section{Desarrollo y Validación de un Método Voltamperométrico para Determinar Trazas de Hierro en Aguas y Otras Matrices}

\section{Resumen}

Se desarrolló una metodología analítica sensible para determinar hierro en muestras de agua y otras matrices. Se utilizó la técnica voltamperométrica de redisolución catódica con pulso diferencial y preconcentración adsortiva utilizando KSCN como ligando y $\mathrm{NaNO}_{2}$ como agente catalítico. El método tiene un ámbito lineal entre $0.7 \mu \mathrm{g} / \mathrm{L}$ y 10.0 $\mu \mathrm{g} / \mathrm{L}$, con un límite de detección de $0.2 \mu \mathrm{g} / \mathrm{L}$ y de cuantificación de $0.7 \mu \mathrm{g} / \mathrm{L}$. La sensibilidad promedio es de $344 \mathrm{nA} /(\mu \mathrm{g} / \mathrm{L})$. La veracidad como $102 \%$ de recuperación para $1.0 \mu \mathrm{g} / \mathrm{L}$ con un coeficiente de variación de $4 \%$. También se estudió la linealidad del sistema Fe-catecol, el cual tiene sensibilidad promedio de $4 \mathrm{nA} /(\mu \mathrm{g} / \mathrm{L})$ menor que el sistema Fe-KSCN y un ámbito lineal mayor, desde $2.4 \mu \mathrm{g} / \mathrm{L}$ hasta $50.0 \mu \mathrm{g} / \mathrm{L}$, un límite de detección de $0.7 \mu \mathrm{g} / \mathrm{L}$ y de cuantificación de $2.4 \mu \mathrm{g} / \mathrm{L}$ con un coeficiente de variación de $8 \%$ y recuperaciones cercanas al $100 \%$ a varios niveles de concentración.

Palabras claves: voltamperometría adsortiva, hierro, tiocianato, nitrito, catecol, análisis de trazas.

\section{Referencias}

1. L. Arroyo, A.L. Alvarado, S. Bravo de Maroto, Ingeniería y Ciencia Química 16-2 (1996) 80.

2. K. Saborío, A.L. Alvarado, S. Bravo de Maroto, Ingeniería y Ciencia Química 15-1 (1995) 4.

3. J. Wang, Stripping Analysis, Principles, Instrumentation and Applications, VCH Publishers, 1985.

4. C.M.G. Van der Berg, Z.Q. Huang, J. Electroanal. Chem. 177 (1984) 269.

5. C.M.G. Van der Berg, M. Nimmo, O. Abollino, E. Mentasi, Electroanalysis 3 (1991) 477. 
6. Z. Gao, K.S. Siow, Talanta 43 (1996) 727.

7. J. Wang, S. Mannino, Analyst 114 (1989) 643.

8. Z. Zhao, W. Jin, J. Electroanal. Chem. 267 (1989) 271.

9. M.G. Paneli, A. Voulgaropoulos, Electroanalysis 5 (1993) 355.

10. J.K. Taylor, Quality Assurance of Chemical Measurements, Lewis Publishers, Inc. 1987. 